\title{
Los instrumentos de gestión de las fronteras exteriores de la Unión Europea ante los flujos migratorios masivos
}

\author{
Management instruments of the external borders of the European \\ Union with respect to the massive migration flows
}

Rafael Pedro MARTínEZ SÁNCHEZ

Doctor en Derecho Internacional

Universidad de Murcia

rafct@hotmail.com

Resumen: El presente artículo se centra en el análisis de los mecanismos de la Unión Europea para el control de sus fronteras exteriores, a la luz de la última gran crisis migratoria y de refugiados que sufre el continente europeo, con especial referencia a la reciente creación de la Guardia Europea de Fronteras y Costas, lo que podría considerarse como una policía fronteriza común europea.

Palabras clave: Crisis migratoria; fronteras exteriores; policía fronteriza común europea.
Abstract: This article focuses on the analysis of EU mechanisms to control its external borders, in the light of the last great migratory and refugee crisis afflicting the European continent, with special reference to the recent creation of the European Border and Coast Guard, what it could be considered as a common European border police.

Keywords: Migratory crisis; external borders; common European border police.

Sumario: I. LA ÚLTIMA GRAN CRISIS MIGRATORIA EN EUROPA. II. EL MARCO JURÍDICO DEL CONTROL DE LAS FRONTERAS EXTERIORES EN LA UE: 2.1. Origen de la gestión común. 2.2. Marco jurídico actual. 2.3. Hacia una europeización de la gestión. III. LOS INSTRUMENTOS DE GESTIÓN DE LAS FRONTERAS EXTERIORES DE LA UE: 3.1. Sistema de Información Schengen (SIS). 3.2. Sistema de Visados (VIS). 3.3. Sistema de impresiones dactilares de solicitantes de asilo (EURODAC). 3.4. ¿Una policía fronteriza común? IV. CONCLUSIÓN.

\section{LA ÚLTIMA GRAN CRISIS MIGRATORIA EN EUROPA}

《 a crisis cuestiona la zona Schengen; los riesgos de seguridad son evidentes tras los ataques de París... el control de las fronteras exteriores debe reforzarse para salvar Schengen» de esta forma se expresaba Frans Timmermans (Vicepresidente de la Comisión), en diciembre de 2015, ante el Parlamento europeo, en relación con la última gran crisis migratoria procedente de Oriente, revelando la gran preocupación generada en Europa.

Como bien sabemos en España, el periodo estival es aprovechado por los inmigrantes y, sobre todo por las redes que los controlan, para poner en marcha la maquinaria de la inmigración irregular. El verano de 2015 trajo consigo 
una avalancha de dimensiones desconocidas para la Unión Europea y que, aunque no con ese volumen, se mantiene a día de hoy. En esta ocasión, la ruta principal seguida por los inmigrantes tiene su origen en la frontera entre Grecia y Turquía, remonta los Balcanes y siguiendo el curso natural del Danubio tiene como destino final Alemania. También los países nórdicos, como Suecia y Finlandia, están siendo destino prioritario. La desestabilización de Siria asolada por una guerra civil desde el año $2011^{1}$ ha sido, sin lugar a dudas, el factor principal que ha provocado esta crisis, como se extrae de los datos ofrecidos por ACNUR en los que se refleja que más de la mitad del total de los que han llegado a Europa son nacionales de ese país.

Es un hecho que la presión migratoria sobre las fronteras europeas no es nueva, sino una constante en las últimas décadas; la cercanía con el continente africano y Oriente medio, hace que Europa sea el objetivo de aquellos que buscan un futuro mejor o huyen de sus países por diversos motivos. Por ejemplo, las revueltas árabes iniciadas en la primavera del año $2010^{2}$ provocaron una importante afluencia de inmigrantes procedentes del sur del Mediterráneo que entraron de manera irregular en la Unión, alcanzando las costas de Italia y Malta. En aquella ocasión, la UE también se vio en la necesidad de adoptar medidas de emergencia, en términos humanitarios y de seguridad, para responder a esta situación, si bien también se pusieron de relevancia sus límites en materia de inmigración y control de fronteras.

Desde otro punto de vista, muchas han sido las voces que han relacionado esta avalancha migratoria con el fenómeno terrorista, a la vez que otras la desvinculan de forma absoluta. Que los flujos migratorios tienen como causa el estado de necesidad de muchos miles de individuos parece evidente, pero

1 Sobre el origen del conflicto de Siria y sus implicaciones: LópeZ-JaCOISTE DíAz, E., «La guerra en Siria y las paradojas de la comunidad internacional, Revista UNISCI, $\mathrm{n}^{\circ} 37$ (enero 2015), pp. 73-97, disponible en <www.ucm.es> [fecha de consulta: 19.07.2016]; GuTIÉRREZ EsPaDA, C., «El conflicto en Siria (2011-2014) a la luz del Derecho Internacional y de la (geo) política, Revista UNISCI, n ${ }^{\circ} 37$ (enero 2015), pp. 99-131, disponible en <www.ucm.es> [fecha de consulta: 19-07-2016]; BERMEJO GARCía, R., «Las conflictivas relaciones de Siria e Israel en el ajedrez del Próximo Oriente», Revista UNISCI, n 37 (enero 2015), pp. 133-146, disponible en <www.ucm.es> [fecha de consulta: 19.07.2016] y LABORIE IGLESIAS, M. A., «Siria: de guerra civil a conflicto regional», Panorama Geopolítico de los conflictos, Instituto Español de Estudios Estratégicos, 2014, pp. 117-147, disponible en <https://dialnet.unirioja.es> [fecha de consulta: 15.07.2016].

2 Sobre la respuesta de la UE ante aquellos hechos, se puede consultar: CERvell HorTaL, M. J., «La UE ante el lado más oscuro de la primavera árabe», Instituto Español de Estudios Estratégicos, $\mathrm{n}^{\circ} 30$ (27.3.2014), disponible en <www.ieee.es> [fecha de consulta: 19.07.2016]. 
que este flujo masivo supone una oportunidad para que las organizaciones terroristas introduzcan a sus miembros en suelo europeo, por más que sean casos aislados, también lo es.

Según se refleja en el «Informe de Situación y Tendencias del Terrorismo de EUROPOL del año 2016 ${ }^{3} \gg$ todavía no existen evidencias que revelen la utilización sistemática de los flujos migratorios para infiltrar terroristas en suelo europeo, pero sí han tenido lugar incidentes terroristas en los que sus autores han hecho uso de aquellos. Por ejemplo, en las investigaciones de los ataques del 13 de noviembre de 2015 en París se constató que dos de los implicados habían entrado en la Unión a través de Grecia entre un gran grupo de inmigrantes procedentes de Siria. Las agencias de información e inteligencia antiterrorista de los Estados miembros con más experiencia en la materia lo tienen más claro, ya que al parecer podrían estar controlando a potenciales terroristas.

Los países más afectados en la Unión Europea respondieron con el restablecimiento temporal de los controles en sus fronteras interiores, acogiéndose a las posibilidades que ofrecen los artículos 23 a 25 del denominado Código de Fronteras Schengen ${ }^{4}$. En principio, el restablecimiento de los controles debía tener una duración máxima total de ocho meses, pero el 12 de mayo de 2016 el Consejo adoptó una Recomendación que permitía mantener los controles temporales en las fronteras interiores en circunstancias excepcionales.

Ante este panorama, la Unión Europea en el contexto de la consecución de un «Espacio de Libertad, Seguridad y Justicia» (ELSJ), a cuyo servicio está el acervo Schengen, cuenta con diversas herramientas, fundamentalmente en forma de bases de datos de control migratorio y agencias, la mayoría de las cuales serán analizadas en este artículo. Destaca, en cuanto a las primeras, el Sistema de Información Schengen (SIS) cuya solvencia ha quedado demostrada desde su puesta en marcha. En lo que respecta a las segundas, la agencia FRONTEX ha sido la referencia en esta materia, siendo recientemente sustituida o englobada en la «Guardia Europea de Fronteras y Costas» con la intención de constituir una especie de policía fronteriza común que se regirá

3 Informe de situación y tendencias del terrorismo de EUROPOL (TE-SAT, Terrorism situation and trend report), 2016, p. 7.

4 Reglamento (CE) 562/2006 del Parlamento Europeo y del Consejo, de 15 de marzo de 2006, por el que se establece un Código comunitario de normas para el cruce de personas por las fronteras, DOUE L 105/1, de 13.04.2006. 
mediante un Reglamento aprobado en septiembre y que entró en vigor el 6 de octubre del año $2016^{5}$.

Un aspecto ciertamente polémico que indica que la solución de la crisis no solo se confía a los instrumentos de ámbito interno, sino a una proyección exterior de la UE ha sido el acuerdo entre la Unión Europea y Turquía, concretado en un «Plan de Acción Conjunto», puesto en marcha el 29 de noviembre de 2015. Como medida más destacada, el 7 de marzo de 2016, Turquía se comprometió al retorno rápido de todos los inmigrantes que no precisaran de protección internacional y que hubieran pasado de Turquía a Grecia, además de la aceptación de todos los migrantes irregulares interceptados en aguas turcas.

Por tanto, el papel de la Unión Europea en las crisis migratorias es creciente, ya que como sucede con respecto a otros fenómenos de dimensiones transnacionales, ha desbordado la respuesta en solitario de los países que la conforman, reclamando una respuesta común europea.

\section{El MARCO JURÍDICO DEL CONTROL DE LAS FRONTERAS EXTERIORES EN LA UNIÓN EUROPEA}

\subsection{Origen de la gestión común}

La necesidad de control de las fronteras exteriores y, en general de una política migratoria común, es una consecuencia necesaria del primigenio objetivo de la consecución de un espacio económico europeo sin fronteras interiores, planteado desde los albores de la creación de una organización supranacional europea. Esta hábil maniobra para la integración europea a través de la integración económica conllevaba para su sostenibilidad la adopción de normas comunes relativas al cruce de las fronteras exteriores y unos principios básicos en materia de inmigración, asilo y visados. El hecho de tener que eliminar los controles fronterizos fue el motivo por el cual los países europeos se vieron abocados a reforzar y progresar en la cooperación; sin embargo, en

5 Reglamento del Parlamento Europeo y del Consejo sobre la Guardia Europea de Fronteras y Costas, de 13.09.2016, por el que se modifica el Reglamento (UE) 2016/399 del Parlamento Europeo y del Consejo y por el que se derogan el Reglamento (CE) no 863/2007 del Parlamento Europeo y del Consejo, el Reglamento (CE) nº 2007/2004 del Consejo y la Decisión 2005/267/ CE del Consejo, disponible en <www.consilium.europa.eu/es/press/press-releases $>$ [fecha de consulta: 14.09 .3026$]$. 
aquellos momentos, esa apuesta por crear un mercado interior no fue acompañada de una atribución de competencias a la Comunidad Europea, por lo que se articularon estructuras de cooperación intergubernamental.

El establecimiento del Acta Única Europea (AUE) -firmada en Luxemburgo el 17 de febrero de 1986- y la firma de los Acuerdos de Schengen el 14 de junio de 1985 y su Convenio de Aplicación (CAAS), de diecinueve de junio de 1990 (mucho más detallado y preciso) influyeron de forma muy positiva en el desarrollo de este mercado interior, sembrando definitivamente la necesidad de la gestión común de las fronteras exteriores y el avance en otras materias de justicia e interior.

Una vez superado el objetivo inicial de un espacio puramente económico, con el Tratado de Maastricht (1992) se apuntó al objetivo de creación de un verdadero espacio de cooperación en asuntos de justicia e interior (incluida la gestión de las fronteras). Pero fue con el Tratado de Amsterdam ${ }^{6}$ (1997) cuando los acuerdos de Schengen fueron incorporados al acervo comunitario, apareciendo por primera vez, de forma expresa, el concepto de «Espacio de Libertad, Seguridad y Justicia» con el objetivo real de comenzar a caminar por la senda de una cooperación más estrecha en justicia e interior, e incluso hacer vislumbrar en el horizonte la posibilidad de llegar a ciertos niveles de integración en estas materias (art. 3, apartado 2, del Tratado de la Unión Europea). El primer hito político para la gestión conjunta de las fronteras exteriores se encuentra en las Conclusiones de la Presidencia del Consejo Europeo, reunido en Tampere, los días 15 y 16 de octubre del año 1999, en las que el Consejo apuntó al fortalecimiento de la cooperación y la asistencia técnica mutua entre los servicios de control fronterizo de los Estados miembros ${ }^{7}$. Posteriormente, en la reunión del Consejo de Justicia, Asuntos de Interior y Protección Civil, de 30 de noviembre y 1 de diciembre de 2000 se hizo una referencia expresa al fortalecimiento del control de las fronteras exteriores en clave de seguridad, mencionando expresamente que se estudiaría la posibilidad de ofrecer a los Estados miembros medios técnicos y personales especializados para aumentar el nivel de eficacia de los sistemas de vigilancia y control de las fronteras exteriores de la Unión más afectadas por los flujos de inmigración ilegal ${ }^{8}$.

6 Sobre el Tratado de Ámsterdam y la cooperación en asuntos de justicia e interior: LEJUENE, Y. (dir.), Le Traitéd'Amsterdam. Espoirs et déceptions, Bruylant, Bruselas, 1998.

Párrafo 24 de la Conclusiones de Tampere, epígrafe IV, apartado A.

8 Sesión n ${ }^{\circ} 2314$ del Consejo de Justicia, Asuntos de Interior y Protección Civil, Bruselas, 30 de noviembre y 1 de diciembre de 2000, epígrafe sobre cooperación entre Estados miembros en la lucha contras las organizaciones de inmigración ilegal, párrafo 3, disponible en <http://europa. eu/rapid> [fecha de consulta: 06.08.2016]. 
El concepto de frontera exterior se fue configurando como un elemento clave para la supervivencia de ese espacio, tal y como se recoge en el Plan para la Gestión de las Fronteras Exteriores de los Estados Miembros de la UE'. La concreción de los diversos aspectos de la gestión de las fronteras exteriores fue llevada a cabo por los programas de Tampere (1999-2004), La Haya (2004-2009) y Estocolmo (2010-2015). En el Programa de Estocolmo (2010-2015) ${ }^{10}$ se hacía balance de los logros obtenidos en esta materia durante los periodos anteriores, destacando la definitiva supresión de las fronteras interiores en el espacio Schengen, la «mejora» en la coherencia de la gestión de las fronteras exteriores de la Unión y el desarrollo de un «Enfoque Global de la Migración».

En la Estrategia de Seguridad Interior de la $U E,{ }^{11}$ como uno de los pilares básicos, se recoge la idea del refuerzo de la seguridad a través de la gestión de las fronteras exteriores; proponiendo que la mejora de la gestión se articule en torno a tres aspectos:

1) Mayor uso de las nuevas tecnologías en los controles fronterizos (segunda generación del Sistema de Información de Schengen (SIS II), Sistema de Información de Visados (VIS), sistema de entrada/salida y programa de registro de pasajeros).

2) Mayor uso de las nuevas tecnologías en la vigilancia de fronteras (Sistema Europeo de Vigilancia de Fronteras, EUROSUR) con el apoyo de los servicios de seguridad GMES ${ }^{12}$ y la creación gradual de un entorno común de intercambio de información sobre cuestiones marítimas de la $\mathrm{UE}^{13}$. En

9 Plan para la Gestión de las Fronteras Exteriores de los Estados Miembros de la UE, Documento del Consejo 10019/02, 13/06/2002, apartado I.1., p. 3.

10 Programa de Estocolmo - Una Europa abierta y segura que sirva y proteja al ciudadano, DO C 115, de 04.05.2010.

11 Comunicación de la Comisión al Parlamento Europeo y al Consejo La Estrategia de Seguridad Interior de la UE en acción: cinco medidas para una Europa más segura [COM (2010) 673 final de 22.11.2010]

$12 \mathrm{El}$ sistema «Global Monitoring for Environment and Security» fue una iniciativa conjunta de la Comisión Europea y la Agencia Espacial Europea que tuvo por objeto desarrollar la capacidad europea de observación autónoma y operativa del la Tierra. En la actualidad ese nombre ha sido sustituido por el de programa «Copernicus» que proporciona información y herramientas para la observación de la superficie terrestre, el mar y la atmosfera, con una finalidad medioambiental y de seguridad, y dentro de esta última para en lo que respecta al control de las fronteras marítimas y terrestres. Sobre GMES se puede consultar: «Application of surveillance tools to Border Surveillance, Concept of Operations», de 7-07-2011, p. 6, disponible en <https://ec.europa.eu> [fecha de consulta: 17.09.16]; y sobre «Copernicus» la página web del programa europeo <www. copernicus.eu/> [fecha de consulta: 17.09.16].

13 Comunicación de la Comisión «Hacia la integración de la vigilancia marítima: Entorno común de intercambio de información sobre cuestiones marítimas de la UE», COM (2009) 538. 
cuanto a las fronteras marítimas de la UE cabe decir que la articulación de toda la seguridad en torno a estas no aparece conectada, directamente, con todas las formas de inmigración ilegal, sino sólo con aquéllas relacionadas con el tráfico clandestino como expresión de la amenaza que supone la delincuencia organizada.

3) Mayor coordinación de los Estados miembros a través de FRONTEX.

La subsiguiente adopción de una serie de actos normativos y no normativos en base a esas orientaciones delimitó la situación actual de la gestión de las fronteras exteriores.

\subsection{Marco jurídico actual}

El Tratado de Lisboa (vigente desde 2009), aunque no supuso un cambio radical en la configuración del ELSJ, ya que prevé un régimen transitorio, lo rediseñó, aportando mayor claridad y sistematización. Este «espacio» se incluye tempranamente y de forma casi idéntica a la recogida en el Tratado de Amsterdam entre los objetivos de la UE, con mención expresa al control de las fronteras exteriores, asilo e inmigración (párrafo $2^{\circ}$ del artículo 3 del Tratado de la Unión Europea):

«La Unión ofrecerá a sus ciudadanos un espacio de libertad, seguridad y justicia sin fronteras interiores, en el que esté garantizada la libre circulación de personas, conjuntamente con medidas adecuadas en materia de control de las fronteras exteriores, asilo, inmigración y de prevención y lucha contra la delincuencia».

En lo que se refiere a la estructura general de este espacio público europeo (Tít. V del Tratado de Funcionamiento de la Unión Europea) se identifican tres componentes básicos, descritos en los párrafos 2 a 4 del artículo 67; libertad, seguridad y justicia, cada uno con sus objetivos políticos propios. Dentro del componente libertad se incluyen una serie de objetivos políticos entre los que se encuentra el desarrollo de una política común de inmigración, asilo y control de las fronteras exteriores, basada en la solidaridad entre Estados miembros y la equidad respecto de los nacionales de terceros países y cuyas concreciones en diversas herramientas serán analizadas.

Es en la gestión de las fronteras exteriores donde los componentes libertad y seguridad se vinculan; el control eficiente de las fronteras exteriores es necesario para garantizar la seguridad dentro del mismo y así poder hacer uso 
de las libertades. El modelo europeo de fronteras ${ }^{14}$ se caracteriza jurídicamente, en primer lugar, por estar configurado como una competencia compartida, $y$, en segundo lugar, por haberse establecido un régimen común de categorías funcionales de frontera: las fronteras interiores y las fronteras exteriores. La definición de fronteras exteriores se recoge en el artículo 1 del Convenio de Aplicación del Acuerdo Schengen (CAAS):

«Las fronteras terrestres y marítimas, así como los aeropuertos y puertos marítimos de las Partes contratantes, siempre que no sean fronteras interiores».

En los artículos 77, 78, 79 y 80, del capítulo II del Título V del Tratado de Funcionamiento de la Unión Europea (TFUE) se establecen los principios sobre los que deben desarrollarse las «políticas sobre controles en las fronteras, asilo e inmigración». Concretamente, en el artículo 77 del TFUE, apartado 1, se establece que:

«La Unión desarrollará una política que tendrá por objetivo garantizar los controles de las personas y la vigilancia eficaz en el cruce de las fronteras exteriores, así como la instauración progresiva de un sistema integrado de gestión de las fronteras exteriores».

En el mismo artículo, en su apartado 2, se prevé el procedimiento legislativo ordinario para que el Parlamento Europeo y el Consejo adopten las disposiciones oportunas en relación con el desarrollo legislativo de los objetivos anteriores.

Por otro lado, las normas para el cruce de personas por las fronteras se compilaron en el denominado Código de fronteras Schengen, mediante la adopción del Reglamento (CE) n $n^{\circ}$ 62/2006 del Parlamento Europeo y del Consejo, de 15 de marzo de $2006^{15}$, modificado recientemente como se expone más adelante. Dicho Reglamento tuvo por objeto consolidar y desarrollar el aspecto legislativo de la política de la Unión en la gestión integrada de las fronteras, detallando las normas relativas al control fronterizo de las personas que crucen sus fronteras exteriores y la citada posibilidad de la reintroducción temporal del

14 Del Valle Gálvez, A., «Las fronteras de la Unión. El modelo europeo de fronteras», Revista de Derecho Comunitario Europeo, $\mathrm{n}^{\circ} 12$ (mayo-agosto 2002), p. 320.

15 Reglamento (CE) n ${ }^{\circ}$ 562/2006 del Parlamento Europeo y del Consejo, de 15 de marzo de 2006, DO L 105 de 13.04 .2006 
control fronterizo en las fronteras interiores. Se considera relevante destacar que cualquier medida de la Unión en materia de cruce de fronteras exteriores e interiores «deben tener en cuenta las disposiciones del acervo de Schengen integrado en el marco de la Unión y, en particular, las disposiciones pertinentes del Convenio de aplicación del Acuerdo de Schengen», tal y como indica el cuarto considerando del Código de Fronteras Schengen.

Para finalizar de dibujar los contornos del régimen jurídico general de la gestión de las fronteras exteriores de la UE, se considera importante resaltar la creciente importancia de la acción exterior europea; en al artículo 21, párrafo 2, letra c del Título V del Tratado de la Unión Europea (TUE) se incluyen de forma expresa «las fronteras exteriores» como objetivo de la acción exterior de la Unión. Ejemplo de lo cual, es el acuerdo expuesto de la UE con Turquía.

\subsection{Hacia una europeización de la gestión}

La continua afluencia de inmigrantes y refugiados a las fronteras exteriores de la Unión Europea ha puesto de manifiesto la imposibilidad de su gestión eficaz desde el prisma estatal. Los conflictos en Oriente Próximo y Medio (Siria e Irak), la situación de caos reinante en Libia ${ }^{16}$ y la inestabilidad política y económica del continente africano en general, seguirán alimentando los flujos migratorios sin control de los que se beneficia la industria de traficantes de personas, en muchas ocasiones relacionadas o formando parte de organizaciones criminales dedicadas también al tráfico de armas, drogas o terrorismo. También, aunque con menos intensidad, en el propio continente europeo se producen flujos migratorios irregulares, como el procedente de los Balcanes.

Actualmente, se identifican cinco rutas principales de acceso a Europa: 1) África occidental, hacia las Islas Canarias, 2) Mediterráneo occidental, hacia el sur de España 3) Mediterráneo central hacia Italia (aprovechando islas de soberanía italiana como Lampedusa), 3) Mediterráneo oriental, la utilizada por los inmigrantes y refugiados de Oriente próximo y medio 4) Mar Negro y 5)

16 Sobre el origen de la inestabilidad en Libia, se puede consultar: BERMEjo GarCía, R., «La protección de la población civil en Libia como coartada para derrocar un gobierno: un mal inicio para la responsabilidad de proteger», Anuario Español de Derecho Internacional, vol. 27, 2011, pp. 9-55. Asimismo, GutiéRreZ EsPaDA, C., «Sobre el 'núcleo duro' de la resolución 1973 (2011) del Consejo de Seguridad y acerca de su aplicación en la práctica», Anuario Español de Derecho Internacional, vol. 27, 2011, pp. 57 a 75 y LóPEZ-JaCOISTE DíAz, E., «La crisis libia desde la perspectiva de la responsabilidad de proteger», Anuario Español de Derecho Internacional, vol. 27, 2011, pp.109-152. 
este de Europa ${ }^{17}$. Esta presión ha ido obligando a los Estados miembros y a la propia Unión Europea a establecer un sustrato común con la progresiva creación de una legislación europea en diversos aspectos que afectan a las fronteras exteriores y en general al fenómeno de migratorio.

Los hitos más destacados son la modernización del Sistema de Información Schengen, medidas sobre el cruce de las fronteras exteriores, la política común en visados y asilo, la política común de inmigración y las relaciones con terceros países en relación con estas materias.

Respecto de la gestión de las fronteras exteriores, varias han sido las medidas adoptadas, destacando la creación de un fondo europeo para ayudar en su financiación ${ }^{18}$, la regulación del «tráfico fronterizo menor ${ }^{19} »$, acciones específicas para el refuerzo de la gestión de la frontera marítima meridional de la $\mathrm{UE}^{20}$, los equipos de intervención rápida en las fronteras $(\mathrm{RABIT})^{21}$, la creación y desarrollo de la agencia FRONTEX, la obligación de los transportistas

17 Según datos facilitados por EUROPOL, aunque la vía del Mediterráneo oriental ha registrado un gran aumento en el último año, la más utilizada por los inmigrantes continúa siendo la central con origen en las costas de Libia, Informe Europol Migration Trend, edición especial, 10.09.2016, disponible en <www.europol.europa.eu> [fecha de consulta: 18.9.2016].

18 Decisión no 574/2007/CE del Parlamento Europeo y del Consejo, de 23 de mayo de 2007, relativa al Fondo para las Fronteras Exteriores para el período 2007-2013 como parte del Programa general «Solidaridad y Gestión de los Flujos Migratorios», establecido mediante Comunicación de la Comisión al Consejo y al Parlamento Europeo por la que se establece un Programa marco de solidaridad y gestión de los flujos migratorios para el período 2007-2013 [COM (2005) 123 final - no publicada en el Diario Oficial].

19 Reglamento (CE) no1931/2006 del Parlamento Europeo y del Consejo, de 20 de diciembre de 2006, por el que se establecen normas relativas al tráfico fronterizo menor en las fronteras terrestres exteriores de los Estados miembros y por el que se modifican las disposiciones del Convenio de Schengen. Se permite la conclusión de acuerdos bilaterales destinados a que los residentes fronterizos puedan beneficiarse de una serie de ventajas cuando crucen las fronteras terrestres exteriores de los países de la UE. Se instituye también un permiso especial para los residentes fronterizos, incluidos aquellos sujetos a la obligación de visado, DO L 405 , 31.12.2006.

20 Comunicación de la Comisión: Refuerzo de la gestión de las fronteras marítimas meridionales de la Unión Europea [COM (2006) 733 final - no publicada en el Diario Oficial].

21 Reglamento (CE) no 863/2007 del Parlamento Europeo y del Consejo, de 11 de julio de 2007, por el que se establece un mecanismo para la creación de equipos de intervención rápida en las fronteras, DO L 199 de 31.7.2007 que modifica el Reglamento (CE) nº 2007/2004 del Consejo por lo que respecta a este mecanismo y regula las funciones y competencias de los agentes invitados. Se establece un mecanismo destinado a facilitar asistencia técnica y operativa reforzada, por un período limitado, en forma de equipos de intervención rápida en las fronteras, incluyendo guardias de fronteras de otros Estados miembros. Estos equipos intervendrán a petición de un Estado miembro que se enfrente a situaciones urgentes y excepcionales resultantes de una gran afluencia de inmigrantes clandestinos. 
aéreos de comunicar los datos sobre pasajeros ${ }^{22}$, la inclusión de la gestión de las fronteras exteriores en la Estrategia de Seguridad Interior de la UE como uno de los puntos esenciales para conseguir un alto grado de seguridad, implantación del «Sistema Europeo de Vigilancia de Fronteras» (EUROSUR) ${ }^{23}$ y la elaboración y aprobación del referido Código de Fronteras Schengen. El objetivo final en esta materia es llegar a alcanzar el concepto de fronteras inteligentes (smart borders), que implica un paquete de medidas destinadas a modernizar la gestión de las fronteras mediante la automatización de los controles fronterizos y la mejora de la información de entrada y salida.

En octubre de 2011, la Comisión presentó una Comunicación sobre fronteras inteligentes ${ }^{24}$, seguida de una propuesta legislativa en febrero de $2013^{25}$. Este paquete estaba compuesto por dos componentes: un Sistema de Entradas y Salidas (SES), a saber, una base de datos para registrar el momento y el lugar de entrada y la duración de las estancias breves autorizadas, y un Programa de Registro de Viajeros (RTP), para simplificar y automatizar los controles fronterizos de ciertas categorías de viajeros, como los que viajan por negocios.

22 Directiva 2004/82/CE del Consejo, de 29 de abril de 2004, sobre la obligación de los transportistas de comunicar los datos de las personas transportadas, DO L 261 de 06.08.2004. Establece que los transportistas aéreos deben recoger y transmitir a las autoridades del Estado miembro de destino, encargadas del control fronterizo, los datos sobre sus pasajeros. En caso de incumplimiento, se les podrá imponer multas o, incluso, en caso de grave violación, la incautación del medio de transporte o la retirada de la autorización de explotación.

23 Reglamento (UE) número 1052/2013 del Parlamento Europeo y del Consejo, de 22 de octubre de 2013, por el que se crea un Sistema Europeo de Vigilancia de Fronteras (Eurosur), DO L 295 de 6.11.2013. Eurosur es un sistema multipropósito destinado a evitar la inmigración ilegal y la delincuencia transfronteriza en las fronteras exteriores. Además, contribuye a garantizar la protección de los inmigrantes que intentan llegar a las costas europeas y a salvar sus vidas. Prevé un mecanismo que permite a las agencias de vigilancia de las fronteras intercambiar rápidamente información y colaborar. Mediante centros nacionales de coordinación, todas las autoridades nacionales de los países de la UE competentes en materia de vigilancia de las fronteras (por ejemplo, guardias de frontera, policía, guardia costera, marina, etc.) deben coordinar sus actividades con las de otros países de la UE y la agencia para la gestión de las fronteras Frontex.

24 Comunicación de la Comisión al Parlamento Europeo y al Consejo: Fronteras inteligentes: opciones y camino a seguir, COM (2011) 680 final, de 25.10.2011.

25 Tres propuestas: 1) Reglamento del Parlamento Europeo y del Consejo por el que se establece un Sistema de Entrada/Salida (EES) para registrar los datos de entrada y salida de los nacionales de terceros países que cruzan las fronteras exteriores de los Estados miembros de la Unión Europea, COM (2013) 95 final, 2) Propuesta de Reglamento del Parlamento Europeo y del Consejo por el que se establece un Programa de Registro de Viajeros, COM (2013) 97 final y 3) Propuesta de Reglamento del Parlamento Europeo y del Consejo por el que se modifica el Reglamento (CE) n ${ }^{\circ}$ 562/2006 en lo que respecta a la utilización del Sistema de Entrada/Salida (EES) y el Programa de Registro de Viajeros (RTP), COM (2013) 96 final. 
En vista de las dudas relativas a los costes iniciales del paquete sobre fronteras inteligentes de la Comisión, los interrogantes acerca de la viabilidad técnica del sistema y las reservas planteadas en torno a los beneficios del proyecto, la Comisión decidió encargar otro estudio técnico, finalizado en octubre de 2014. En 2015 se llevaron a cabo una serie de pruebas prácticas y operativas bajo los auspicios de la Agencia EU-LISA ${ }^{26}$.

En abril de 2016, la Comisión presentó una nueva propuesta, en esta ocasión únicamente sobre un nuevo $\mathrm{SES}^{27}$ que persigue el mismo objetivo esencial, cual es el de acelerar, facilitar y reforzar los procedimientos de control fronterizo para los viajeros de terceros países, presenta, no obstante, cambios significativos en comparación con la propuesta de 2013. En primer lugar, reduce la cantidad de datos (por ejemplo, las huellas dactilares) que han de recogerse y almacenarse, y propone una notable reducción de los costes, que pasarían de los 1.100 millones de euros iniciales a 408 millones. Además, esta nueva propuesta de la Comisión prevé la interoperabilidad entre el Sistema de Información de Visados (véase más arriba) y el SES, así como el acceso al SES de los organismos con fines coercitivos en determinadas condiciones. Esta propuesta es, en la actualidad, objeto de examen en el Parlamento y el Consejo.

Los últimos impulsos en cuanto a la gestión común de las fronteras exteriores han venido de la mano de esta última crisis migratoria. En primer lugar, el impulso de la creación de una policía fronteriza común (cuya regulación, en proceso de aprobación, se analiza en el apartado III sobre los instrumentos concretos). En segundo lugar, otra media que bien no podría considerarse como un avance pero sí pudiera ser necesaria, es la propuesta de la Comisión, en diciembre de 2015, para la introducción de controles sistemáticos a los ciudadanos de la UE, lo que conlleva la posibilidad de consultar las bases de datos pertinentes de todas las personas que entren en el espacio Schengen o salgan de él, implicando ello la modificación del Código de Fronteras de Schengen.

26 Agencia Europea para la Gestión Operativa de Sistemas Informáticos de Gran Magnitud en el Espacio de Libertad, Seguridad y Justicia, cuya función es la gestión de sistemas informáticos de gran magnitud de la UE, operativa desde 1 de diciembre de 2012.

27 COM (2016) 194 final, de fecha 6.4.2016 de la Comisión mediante la que se hace una propuesta de Reglamento del Parlamento Europeo y del Consejo por el que se establece un Sistema de Entradas y Salidas (SES) para registrar los datos de entrada y salida y de la denegación de entrada de los nacionales de terceros países que cruzan las fronteras exteriores de los Estados miembros de la Unión Europea, se determinan las condiciones de acceso al SES con fines coercitivos y se modifican el Reglamento (CE) nº 767/2008 y el Reglamento (UE) nº 1077/2011. 
Como consecuencia se aprobó el Reglamento (UE) 2016/399 del Parlamento Europeo y del Consejo, de 9 de marzo de 2016, por el que se establece un Código de normas de la Unión para el cruce de personas por las fronteras (se modifica el Código de fronteras Schengen) ${ }^{28}$. El establecimiento de controles obligatorios para los ciudadanos de la Unión que entran o salen del espacio Schengen por cualquier vía fue concebido tras los atentados de París de noviembre de 2015 para reforzar la seguridad en caso de agravamiento de la amenaza terrorista, pensando en el fenómeno de los combatientes terroristas extranjeros, muchos de ellos nacionales de los Estados miembros. De acuerdo con esa modificación, se realizarán controles a los ciudadanos europeos usando el SIS y la base de datos de documentos de viaje perdidos o robados de INTERPOL.

Finalmente, otro hecho ciertamente novedoso que también apunta hacia la gestión global de las fronteras europeas se refiere, primero, a la participación de la OTAN en la crisis y, sobretodo, a la celebración de un acuerdo de coordinación entre esta y la $\mathrm{UE}^{29}$.

\section{LOS INSTRUMENTOS DE GESTIÓN DE LAS FRONTERAS EXTERIORES DE LA UNIÓN EUROPEA}

Como se ha expuesto, el primer hito en la gestión común de las fronteras exteriores de la Unión tuvo lugar el 14 de junio de 1985, fecha en la que cinco de un total de diez Estados miembros de la Comunidad Económica Europea firmaron el Acuerdo de Schengen, completado posteriormente (1990) con el Convenio de aplicación del Acuerdo de Schengen. Por esa razón, la mayor parte de los instrumentos que se encargan de la gestión de fronteras en el territorio de la UE se ha gestado al abrigo del acervo Schengen.

\subsection{Sistema de Información Schengen (SIS)}

Si la cooperación Schengen se considera un ejemplo de colaboración asentada y eficaz, el Sistema de Información Schengen (SIS) es su herramienta principal; es la base de datos europea con más tradición y paradigma de las de

\footnotetext{
28 Reglamento (UE) 2016/399 del Parlamento Europeo y del Consejo, de 9 de marzo de 2016, por el que se establece un Código de normas de la Unión para el cruce de personas por las fronteras (Código de fronteras Schengen), DOUE L no 77 de 23.03.2016.

29 Acuerdo de cooperación firmado en Varsovia, sede de FRONTEX, el 6 de marzo de 2016.
} 
nueva creación, existiendo dos versiones del sistema, el SIS I y el SIS II. En el espíritu de su creación están los retos que en cuanto a seguridad se planteaban con la abolición de los controles en las fronteras interiores, con el fin de salvaguardar la seguridad e impedir la inmigración ilegal de ciudadanos de terceros Estados no miembros de las Comunidades Europeas. Es decir, había que adoptar algunas medidas compensatorias.

El SIS tiene como objeto dos finalidades fundamentales: 1) la original, que es la aplicación de las disposiciones de dicho Convenio sobre la circulación de personas por los territorios de las Partes contratantes, con la ayuda de la información transmitida por el SIS con arreglo a lo dispuesto en dicho Convenio (art. 93 del Convenio de Schengen de 1990) y 2) la preservación del orden y la seguridad públicos, incluida la seguridad de los Estados miembros. Por tanto, la función original del SIS era y sigue siendo el control sobre las personas que quieren entrar en el «Espacio Schengen». Inicialmente, fue concebido como un sistema de recopilación de información para llevar a cabo la labor de control fronterizo, pero posteriormente se le añadieron otras funciones relacionadas con la investigación. Con ellas se pretendía dar solución a la urgente necesidad de utilizar el sistema con vistas, también, a la lucha antiterrorista, adelantando además ciertas modificaciones, sin perjuicio de las que traería la implantación del SIS de segunda generación (SIS II) en el año 2013. Debido a esta doble funcionalidad, en sus sucesivas modificaciones y mejoras el SIS ha requerido la adopción de dos actos que afectaban a la aplicación de las disposiciones previstas tanto en materia de circulación de personas (Tít. IV del Tratado de la Comunidad Europea) como a la cooperación judicial en materia penal y policial (Tít. VI del Tratado de la Unión Europea).

La base jurídica del SIS está en el Título IV del Convenio de Aplicación del Acuerdo Schengen (CAAS). Su artículo 92, apartado 1 obliga a las partes contratantes a participar en la creación y mantenimiento del mismo y permite, asimismo, que las autoridades dispongan de un procedimiento de consulta automatizada «al efectuar controles en la frontera y comprobaciones y otros controles de policía y de aduanas realizados dentro del país de conformidad con el derecho nacional». También en ese artículo se recoge la arquitectura $\mathrm{y}$ finalidades técnicas del sistema, que adopta la forma de «estrella», con un sistema central (C-SIS) y unos sistemas nacionales (NSIS). El C-SIS, o «unidad de apoyo técnico», es el elemento central de este engranaje y su cometido principal es el de ofrecer un soporte técnico. Una característica reseñable es que no se pueden limitar los países destinatarios de la información, sino que los datos son de difusión general. Tampoco se pueden enviar señalamientos 
directamente entre Estados, sino sólo a través del C-SIS. Estas características, de hecho, se corresponden con la filosofía general del Convenio, que es la cooperación y colaboración directa entre los Estados de forma que todos tengan el mismo nivel de información.

Las funciones de control que precisa toda base de datos de carácter personal, son ejercidas por una «Autoridad de Control Nacional» -en España es la Agencia de Protección de Datos- y la «Autoridad de Control Común». Todos los países miembros han tenido que dotarse de una legislación de protección de datos que reúna al menos las garantías básicas requeridas a nivel europeo ${ }^{30}$. $\mathrm{Al}$ elaborarse el estudio de viabilidad del SIS se advirtió la necesidad de instituir un servicio en cada Estado, como complemento del ordenador, consistente en unas oficinas con personal que complementaran el frío acceso al sistema informático. Son las denominadas oficinas SIRENE ${ }^{31}$ (Oficinas de solicitud de información complementaria a la entrada nacional), muy útiles para aportar información complementaria relativa a la introducción en el SIS de señalamientos.

Se pueden distinguir dos clases de competencias en la Oficina SIRENE: 1) la competencia original o propia que le confiere el ser el único punto de contacto para el intercambio de información complementaria relativa a los señalamientos en el SIS, y 2) la competencia derivada o concedida, está conferida por las autoridades nacionales para intercambiar información en base a los artículos 39, 40,41, 46 del Convenio de Aplicación del Acuerdo de Schengen y desarrollada en el «Vademécum o Manual de la cooperación policial transfronteriza». En todos los Estados miembros existe una oficina, lo que supone un punto único y de acceso permanente que hace de nexo con el resto de los Estados, especialmente, con el fin de comunicar el descubrimiento de un señalamiento

30 El marco general de la protección de datos personales tratados en el ámbito de la cooperación policial y judicial, para la prevención, la investigación, la detección o el enjuiciamiento de infracciones penales o la ejecución de sanciones penales nos remite a la Decisión Marco 2008/977/JAI del Consejo, de 27 de noviembre de 2008, relativa a la protección de datos personales tratados en el marco de la cooperación policial y judicial en materia penal, DO $\mathrm{n}^{\circ} \mathrm{L} 350$ de 30.12.2008, pp. 60-71.

Se encuentra en proceso de aprobación la actualización y modernización de esta normativa, existiendo una propuesta por parte de la Comisión de una Directiva del Parlamento Europeo y del Consejo relativa a la protección de las personas físicas en lo que respecta al tratamiento de datos personales por las autoridades competentes a efectos de la prevención, investigación, detección y enjuiciamiento de infracciones penales o la ejecución de sanciones penales, y a la libre circulación de estos datos.

31 Proviene del acrónimo inglés Supplementary Information Request at the National Entry. 
y simultáneamente solicitar información sobre los motivos de la inclusión de tal señalamiento en el SIS.

En suma, el primer SIS contenía exclusivamente información sobre personas que pudieran haber estado implicadas en delitos graves o que pudieran no tener derecho a entrar o permanecer en la Unión Europea y descripciones de personas desaparecidas (especialmente niños) así como información sobre vehículos, armas de fuego, billetes de banco y documentos de identidad que hubieran podido ser robados, sustraídos o extraviados. Pero la ampliación de la Unión Europea, las nuevas amenazas y los avances tecnológicos, provocaron que se planteara «dar un salto» en la concepción de esta base de datos. El 9 de abril de 2013, el Sistema de Información de Schengen de segunda generación (SIS II) sustituyó a la anterior versión (SIS I).

Como se ha referido, puesto que en el momento en el que se estaba gestando el sistema de segunda generación, su regulación afectaba a materias de dos pilares (el primero y el tercero), se optó, como solución, por la adopción de dos actos. Esa es la razón por la que la base legislativa del SIS II está compuesta por un Reglamento («Reglamento del SIS II») y una Decisión ( Decisión del SIS II»), compartiendo ambos unas disposiciones comunes, por más que cada uno tenga disposiciones específicas. Las disposiciones específicas que rigen el sistema en lo que respecta a los procedimientos de descripción previstos en el Título IV del Tratado constitutivo de la Comunidad Europea (antiguo primer pilar) están reguladas por el Reglamento (CE) número 1987/2006 del Parlamento Europeo y del Consejo, de 20 de diciembre de 2006, relativo al establecimiento, funcionamiento y utilización del Sistema de Información de Schengen de segunda generación (SISII) ${ }^{32}$. Las disposiciones específicas que rigen su uso con los fines previstos en el Título VI del Tratado de la Unión Europea (antiguo tercer pilar) se recogieron en la Decisión 2007/533/7AI, de 12 de junio de 2007, relativa al establecimiento, funcionamiento y utilización del Sistema de Información de Schengen de segunda generación (SIS II) ${ }^{33}$.

Recientemente se ha adoptado la Decisión de Ejecución (UE) 2016/1345 de la Comisión, de 4 de agosto, sobre las normas minimas de calidad de los datos de los registros de impresiones dactilares en el marco del Sistema de Información de Schengen de segunda generación (SIS II) ${ }^{34}$, con ello se trata de aumentar la efectividad

32 DOUE L 381 de 28.12.2006, pp. 4-23.

33 DOUE L 205 de 7.08.2007, pp. 63-84.

34 DOUE, L 213 de 6.08.2016, pp. 15-20. 
en las identificaciones dactilares de cara a la próxima inclusión de un sistema automático de identificación dactilar (SAID), en el marco del propio SIS II. Cabe destacar que el buen funcionamiento de la identificación dactilar veraz supone un factor clave en la gestión efectiva de las fronteras exteriores, entre otras materias.

En esencia, la virtualidad práctica del sistema consiste en que una autoridad fronteriza, aduanera, judicial o de cualquier otra índole de un Estado miembro puede generar una «alerta» en la que se describa la persona o el objeto que se está buscando, por las razones siguientes: para evitar la entrada de personas que no están autorizadas a entrar o a permanecer en territorio Schengen; para buscar y detener a una persona contra la que se ha dictado una orden de detención europea; para ayudar a localizar personas, previa petición de las autoridades policiales o judiciales; para buscar y proteger a una persona desaparecida y para buscar propiedades robadas o perdidas

La realidad es que el mayor volumen de intercambio de información policial en Europa continúa teniendo lugar a través del SIS para la obtención de datos básicos que figuren en el sistema y de las Oficinas SIRENE que existen en cada Estado para ampliar esa información.

\subsection{Sistema de Información de Visados (VIS)}

También dentro del marco Schengen, con motivo de los ataques del 11 de septiembre de 2001, y para frenar el uso desmedido y fraudulento de los regímenes de visados de los Estados miembros, se convino promover la adopción de una «política común de visados» y poner en marcha con ello el intercambio de información sobre visados de corta duración. Por tanto, el Sistema de Información de Visados (Visa Information System - VIS) está concebido con la idea de dar respuesta a dos aspectos: 1) ayudar a aplicar una política de visados común facilitando el examen de las solicitudes de visado y los controles de las fronteras exteriores y 2) contribuir a prevenir las amenazas a la seguridad interior de los Estados miembros, lo que abre la puerta a su utilización para la investigación policial.

Este segundo objetivo motivó la adopción de la Decisión del Consejo 2008/633/7AI de 23 de junio de 2008 sobre el acceso para consultar el Sistema de Información de Visados (VIS) por las autoridades designadas de los Estados miembros y por la Oficina Europea de Policía (EUROPOL) con fines de prevención, detección e investigación de los delitos de terrorismo y otros delitos graves. El VIS se aplica en todos los Estados miembros (excepto en Irlanda y, de momento, en el Estado 
Miembro, Reino Unido), así como en Suiza, Noruega e Islandia. El VIS tiene como objetivo mejorar la aplicación de la política común de visados, la cooperación consular y la consulta entre las autoridades centrales competentes en materia de visados, por ello el VIS está conectado a todos los consulados de los Estados Schengen encargados de la expedición de visados y a todos los puntos de cruce de las fronteras exteriores.

En estos pasos fronterizos el VIS permite a la policía de fronteras comprobar que el titular de un visado biométrico es efectivamente la misma persona que lo solicitó. Esto es posible gracias a la comparación de las impresiones dactilares tanto con el archivo biométrico vinculado al visado como con todos los datos de la base de datos VIS. Se ha dotado al sistema de unos elevados niveles de seguridad para garantizar su solidez y disponibilidad en todo momento, así como un acceso a los datos reservado, únicamente, al personal autorizado y para los fines autorizados. Esta base informática incluye datos sobre filiación, nacionalidad actual y de origen, tipo y número de documento de viaje, autoridad que lo expidió, fechas de expedición y de expiración, destino principal y duración de la estancia prevista, motivo del viaje, fechas de llegada y de salida previstas, primera frontera de entrada o itinerario de tránsito previstos, residencia, impresiones dactilares, tipo de visado y número de la etiqueta adhesiva de visado, información detallada sobre la persona que ha cursado una invitación o que puede sufragar los gastos de mantenimiento del solicitante durante la estancia. Y, además, hay posibilidad de acceso a las solicitudes de visados, fotografías, impresiones dactilares, decisiones relacionadas de las autoridades responsables de los visados y enlaces entre aplicaciones relacionadas.

\subsection{Sistema de impresiones dactilares de solicitantes de asilo (EURODAC)}

Otra herramienta para la gestión común de fronteras, disponible desde el 20 de julio de 2015, es la base de datos EURODAC que contiene las solicitudes de asilo con las impresiones dactilares correspondientes que se reciben en cualquier punto de la UE. Esta base ha venido demostrando su utilidad desde 1993, año de su introducción. Hasta fechas recientes, este sistema estaba regulado mediante el Reglamento (CE) 2725/2000 del Consejo, de 11 de diciembre del 2000, relativo a la creación del sistema EURODAC para la comparación de las impresiones dactilares en la aplicación efectiva del Convenio de Dublín ${ }^{35}$ y

35 DO L 316 de 15.12.2000. 
desarrollado por el Reglamento (CE) 407/2002 del Consejo, de 28 de febrero del 2002. Pero desde julio de 2015 entró en vigor el Reglamento (UE) 603/2013 del Parlamento Europeo y del Consejo, de 26 de junio de 2013, relativo a la creación del sistema EURODAC para la comparación de las impresiones dactilares para la aplicación efectiva del Reglamento (UE) 604/2013 ${ }^{36}$, por lo que también pasó a ser una herramienta más para la investigación de organizaciones e individuos.

La implementación de esta base de datos se enmarca en la creación del «sistema europeo común de asilo», directamente relacionado con la gestión común de las fronteras exteriores, es uno de los elementos constitutivos en el establecimiento progresivo de un espacio de libertad, seguridad y justicia «abierto» para quienes, obligados por las circunstancias, busquen protección internacional en la Unión. Hasta fechas recientes, este sistema estaba facilitando a las autoridades responsables la gestión de las solicitudes de asilo de los Estados de la Unión Europea la identificación de los solicitantes y de las personas interceptadas en relación con el «cruce irregular» de las fronteras exteriores de la Unión, y ahora, también, permite a los servicios de seguridad, bajo condiciones estrictas, consultar la base de datos con fines de prevención, detección o investigación de los delitos de terrorismo o de delitos graves. En el octavo considerando de este nuevo Reglamento se contempla expresamente que:

«La información que contiene EURODAC es necesaria a efectos de prevención, detección investigación de los delitos de terrorismo como se contempla en la Decisión Marco del Consejo 2002/475/JAI, de 13 de junio de 2002, sobre la lucha contra el terrorismo...».

Con la comparación de los datos lofoscópicos, los países de la UE pueden acreditar si un solicitante de asilo o un extranjero cuya presencia ilegal se ha detectado en su territorio ha solicitado ya el asilo en otro país de la UE, o si un solicitante de asilo ha entrado irregularmente en el territorio de la Unión

36 Reglamento por el que se establecen los criterios y mecanismos de determinación del Estado miembro responsable del examen de una solicitud de protección internacional presentada en uno de los Estados miembros por un nacional de un tercer país o un apátrida, y a las solicitudes de comparación con los datos de EURODAC presentadas por los servicios de seguridad de los Estados miembros y EUROPOL a efectos de aplicación de la ley, y por el que se modifica el Reglamento (UE) 1077/2011, por el que se crea una Agencia europea para la gestión operativa de sistemas informáticos de gran magnitud en el espacio de libertad, seguridad y justicia (refundición) [DO L 180 de 29.6.2013, aplicable desde el 20 de julio de 2015]. 
y ahora; además, posibilita que las fuerzas policiales de cada país y la agencia europea de policía comparen impresiones dactilares vinculadas con investigaciones penales con las que contiene EURODAC ${ }^{37}$. Por tanto, EURODAC y el VIS son herramientas creadas, en principio, para la regulación de la inmigración, el asilo y el control del cruce de fronteras exteriores pero, en vista de su utilidad y de la importancia del objetivo que se persigue, con a mi juicio buen criterio siempre que se cumplan los requisitos legales para ello, se ha posibilitado su uso, también, con el fin de la investigación del crimen organizado y el terrorismo.

\section{4. ¿Una policía fronteriza común?}

Como se ha adelantado, la última crisis migratoria hacia Europa proveniente fundamentalmente de países del Oriente próximo y medio ha provocado que la Agencia Europea para la Gestión de la Cooperación Operativa en las Fronteras Exteriores (FRONTEX) sea absorbida por un organismo europeo con más competencias y medios en la materia, lo que podría suponer un salto cualitativo en la materia.

La gestión del fenómeno ha provocado críticas de los Estados miembros hacia la UE y, a la misma vez, críticas de la UE hacia determinados países como Grecia. Como se ha expuesto, el Tratado de Lisboa prevé la adopción de una política común de gestión de las fronteras y, en el marco del Programa de Estocolmo, se pidió el estudio de la viabilidad de un sistema europeo de guardia de fronteras, petición reiterada en las Conclusiones del Consejo Europeo de junio de 2014. A la vista de la alarmante situación, Berlín y París presionaron para la adopción de medidas urgentes sobre la gestión de fronteras, en especial la creación inmediata de un cuerpo especial de policía fronteriza.

Recogiendo esa preocupación la Comisión Europea propuso la iniciativa el 15 de diciembre de 2015. Tras nuevas críticas y debates, el Consejo, el Parlamento y la Comisión alcanzaron el acuerdo el 21 de junio y al día siguiente, el Comité de Representantes Permanentes (COREPER) confirmó, en nombre del Consejo, el texto transaccional acordado con el Parlamento Europeo

37 Además de las impresiones dactilares, los datos transmitidos por los países de la UE contienen: el país de la UE de origen, el sexo de la persona, el lugar y fecha de la solicitud de asilo o la interceptación de la persona, el número de referencia, la fecha de toma de impresiones dactilares y la fecha de transmisión de datos a la unidad central. 
en relación con la propuesta de un Reglamento sobre la Guardia Europea de Fronteras y Costas. Al fin, el 14 de septiembre de 2016, el Consejo dio su aprobación final a la Guardia Europea de Fronteras y Costas, con la adopción del Reglamento que la regula. Se calcula que esta policía fronteriza común podría estar operativa a mediados de octubre, a partir de que el Reglamento entre en vigor el 6 de octubre de 2016 (veinte días después de su publicación en el Diario Oficial), con la salvedad de las disposiciones relativas a la creación de la reserva y de los equipos técnicos conjuntos que entrarán en vigor dos meses después que el resto del articulado, y las relativas al retorno conjunto tres meses después.

Esta Guardia englobara, por un lado, a la Agencia Europea de Guardia de Fronteras y Costas (partiendo de FRONTEX pero con funciones adicionales) y, por otro, a las autoridades responsables de la gestión de fronteras de los Estados miembros. Su objetivo principal sería garantizar y aplicar, como responsabilidad compartida, la gestión europea integrada de las fronteras exteriores, en dos direcciones: tanto para lograr una administración migratoria efectiva, como para asegurar un elevado nivel de seguridad dentro de la UE. La idea no es la creación de un contingente con base propia, diferente de los cuerpos fronterizos de los Estados, sino que, en caso de emergencia estos serán movilizados y acudirán a misiones temporales, recibiendo formación específica.

Un primer análisis de la regulación revela su extensión que abarca no solo la gestión de las fronteras sino que cubre también, directa o indirectamente otras políticas de la UE, tales como el derecho de los refugiados, la acción exterior en esta materia, la migración y la seguridad, tanto interna como externa. Algunos juristas han considerado que el texto, pese a ser excesivamente ambicioso, no alcanza los objetivos que se propone, estimando asimismo que hunde sus raíces en el viejo modelo intergubernamental europeo ${ }^{38}$.

Se pretende que esa nueva Agencia europea (la que parte de FRONTEX) centre sus actividades en establecer una estrategia operativa en materia de fron-

38 Al respecto: PeERS, S., «The Reform of Frontex: Saving Schengen at Refugees' Expense?», EU Law Analysis, 16.12.2015, disponible en <http://eulawanalysis.blogspot.com.es> [fecha de consulta: 09.07.2016], Carrera, S. y Den Hertog, L., «A European Border and Coast Guard: What's in a name?», Centre for European Policy Studies, $n^{\circ} 88$, marzo (2016), disponible en <www.ceps.eu/> [fecha de consulta: 09.07.2016]; y DE BRUYCKER, P., «The European Border and Coast Guard: A New Model Built on an Old Logic», European Papers, vol. 1(2016), $\mathrm{n}^{\circ} 2$, pp. 559-569, disponible en <www.europeanpapers.eu> [fecha de consulta: 09.07.2016]. 
teras exteriores, ayudando a todos los Estados miembros a aplicarla. A tal fin, los objetivos declarados por las Instituciones europeas serían los siguientes:

- Elaboración de una evaluación de la vulnerabilidad en lo que respecta a la capacidad de control de las fronteras de los Estados miembros;

- Organización de operaciones conjuntas e intervenciones rápidas en las fronteras ${ }^{39}$ para reforzar la capacidad de los Estados miembros de cumplir sus obligaciones por lo que se refiere al control de las fronteras exteriores, y haga frente a los desafíos que se planteen en la frontera exterior como consecuencia de la inmigración ilegal o la delincuencia transfronteriza;

- Ayuda a la Comisión a coordinar los equipos de apoyo para la gestión de las corrientes migratorias cuando un Estado miembro se enfrente a presiones migratorias desproporcionadas en puntos críticos de sus fronteras exteriores;

- Garantías de la ejecución práctica de medidas en las situaciones que exijan una actuación urgente en las fronteras exteriores;

- Prestación de asistencia técnica y operativa para las operaciones de búsqueda y salvamento de personas en peligro en el mar que puedan plantearse durante las operaciones de vigilancia fronteriza en el mar;

- Puesta en común obligatoria de recursos humanos, estableciendo un contingente de intervención rápida integrado por al menos 1.500 guardias de fronteras;

- Nombramiento de funcionarios de enlace de la Agencia en los Estados miembros;

- Organización y coordinación de operaciones de retorno e intervenciones;

- Fomento de la cooperación con terceros países, coordinando la cooperación operativa entre estos y los Estados miembros en la gestión de las fronteras.

Entrando en el análisis del texto final, el Reglamento en su artículo 2 ofrece una serie de definiciones comunes. Entre estas destaca «hotspot area» 0 «punto crítico», una zona en la que el Estado miembro de acogida, la Comisión,

39 Las operaciones conjuntas no son algo nuevo, sino que se venían realizando por FRONTEX con asiduidad y cierto grado de eficacia. La operación conjunta «Tritón», puesta en marcha a finales de 2014 y ampliada sustancialmente en 2015, con la misión de vigilar la frontera marítima principalmente entre Libia e Italia, reúne a personal y equipos de varios Estados miembros para ofrecer a Italia un apoyo operativo de primera línea. En Grecia, la operación conjunta «Poseidón» se amplió considerablemente en diciembre de 2015 para convertirse en una operación de intervención rápida, con un mayor despliegue de personal y equipos técnicos para hacer frente a los desafíos relacionados con la gestión de las fronteras exteriores. 
los organismos pertinentes de la Unión y los Estados miembros participantes cooperan, con el objetivo de gestionar un desafío migratorio desproporcionado existente o potencial, caracterizado por un aumento significativo en el número de inmigrantes que llega la fronteras exteriores. Asimismo, se considera significativo que se definan ciertos conceptos como los de: «retorno», «retornado», «decisión de retorno», «operación de retorno»e «intervención de retorno».

En el artículo 5 se hace hincapié en la responsabilidad compartida entre la Unión y los Estados miembros en lo que a la gestión de las fronteras exteriores se refiere, no se sabe si en un intento de convencer a los Estados miembros de las bondades de esta nueva agencia que tendrá la posibilidad de intervenir en su territorio o con la finalidad de hacer conscientes a estos de la importancia de una gestión común. Para llevar a cabo las labores conferidas a la Agencia, el artículo 10 establece la «obligación de intercambiar información» de forma «puntual y precisa» entre esta y las autoridades nacionales responsables de la gestión de las fronteras y en materia de retorno, incluidos los guardias de costas en la medida en que lleven a cabo tareas de control de fronteras. En el artículo 13 se establece que la Agencia llevará acabo una evaluación de la vulnerabilidad del control de fronteras exteriores por parte de los Estados miembros, centrada en la disponibilidad de equipos técnicos, sistemas, capacidades, recursos, infraestructura y personal adecuadamente cualificado y formado.

Del artículo $14 \mathrm{al} 26$ se regula la intervención de la Agencia en el territorio de un Estado miembro. En el primer párrafo del artículo 14 se recoge que un Estado miembro podrá de forma voluntaria solicitar la asistencia de la Agencia en el cumplimiento de sus obligaciones con respecto al control de las fronteras exteriores, pudiendo tomar una o varias medidas, entre las que destacan:

1. Puesta en marcha de operaciones conjuntas e intervenciones fronterizas rápidas en las fronteras exteriores (arts. del 15 al 17): un Estado miembro podrá pedir a la Agencia que inicie operaciones conjuntas para hacer frente a los retos venideros, como la migración ilegal, las amenazas actuales y futuras en las fronteras exteriores o la delincuencia transfronteriza, o que le preste una mayor asistencia técnica y operativa para el cumplimiento de sus obligaciones de control de las fronteras exteriores.

2. Envío de Equipos de apoyo para la gestión de la migración (art. 18): previsto para los casos en los que un Estado miembro se enfrente a «retos migratorios desproporcionados» en determinados puntos críticos de sus fronteras exteriores debido a grandes afluencias de flujos migratorios mixtos. 
Pero el aspecto más polémico de la nueva regulación es el que permite a la Agencia, si la situación lo requiere, intervenir forzosamente en un Estado miembro. En el referido artículo se ha recogido la que podría considerarse la medida más intrusiva en la soberanía de los Estados miembros, ya que se faculta a la Agencia para poder intervenir en los casos en los que exista una situación en las fronteras exteriores que requiera de una actuación urgente. En el primer apartado se establece que la Agencia podrá actuar de urgencia cuando el control de las fronteras exteriores se haya vuelto «ineficaz» en grado tal que pueda poner en peligro el funcionamiento del espacio Schengen por dos causas: a) porque un Estado miembro no haya atendido a la recomendación conforme al 13.8 o b) a casusa de una situación concreta y desproporcionada y no haya solicitado ayuda suficiente a la Agencia en virtud de los artículos 15, 17 o 18 o bien no esté dando los pasos necesarios para adoptar las medidas establecidas en dichos artículos. En esta tesitura, «el Consejo, a propuesta de la Comisión, podrá adoptar sin demora, mediante un acto de ejecución, una decisión en la que se fijen las medidas que deberá adoptar la Agencia al objeto de aminorar dichos riesgos y en la que se exija al Estado miembro implicado que coopere con la Agencia en su aplicación», imponiendo la obligación de que la Comisión consulte con la Agencia antes de formular su propuesta. En su segundo apartado se contempla que en estos casos, el Parlamento Europeo debe ser informado «sin demora de todas las medidas y decisiones» posteriores adoptadas en respuesta.

En el tercer apartado se ha previsto el catálogo de medidas a adoptar por la Agencia, esto es: la organización y coordinación de la intervención en las fronteras, despliegue de equipos propios de la Guardia europea, coordinación de varios Estados miembros y terceros países, despliegue de equipos técnicos o la organización de operaciones de retorno.

En cuanto a los plazos contemplados para ese despliegue de urgencia, en el cuarto apartado del artículo 19 se refiere que el director ejecutivo de la Agencia determinará las acciones que deban realizarse para la ejecución práctica de las medidas previstas en dicha decisión del Consejo (incluidos los equipos técnicos y el número y los perfiles de los guardias de fronteras y otro personal competente que se requieran para lograr los objetivos de dicha decisión) y elaborará un plan operativo que presentará a los Estados miembros implicados en el plazo de dos días hábiles a partir de la fecha de adopción de la decisión. En el plazo de tres días hábiles a partir de la fecha de presentación del plan operativo, el director ejecutivo y el Estado miembro implicado deberán alcanzar un acuerdo sobre el mismo, según se establece en el apartado quinto. 
Para la ejecución de las medidas previstas en la decisión del Consejo, la Agencia desplegará de inmediato, y en cualquier caso en el plazo de cinco días hábiles a partir de la fecha en que se fije el plan operativo, el personal necesario del contingente de reacción rápida a que se refiere el artículo 20, apartado quinto. Se dispone que en una segunda fase se desplegarán los equipos de la Guardia Europea de Fronteras y Costas adicionales que resulten necesarios, y siempre en el plazo de siete días hábiles a partir de la fecha de despliegue del contingente de reacción rápida. En el apartado octavo se impone la obligación del Estado miembro implicado de ejecutar la decisión del Consejo a que se refiere el apartado primero, cooperando con la Agencia y tomando las medidas necesarias para facilitar la intervención y la ejecución práctica de las medidas fijadas. También, se impone de forma expresa a los Estados miembros el deber de facilitar los guardias de fronteras y demás personal competente, así como personal involucrado en tareas de retorno, que requiera el director ejecutivo según lo previsto en el cuarto apartado del presente artículo (apartado 9 del artículo 19). En el último apartado del artículo 19, el décimo, se dispone una cláusula para el caso en el que el Estado miembro en cuestión no ejecute la decisión del Consejo en un plazo de 30 días y no coopere con la Agencia con arreglo a lo dispuesto en el apartado octavo del presente artículo, advirtiendo de la posibilidad de que la Comisión active el procedimiento previsto en el artículo 29 del Reglamento (UE) 2016/399.

Como se observa, el Reglamento es muy detallado a la hora de establecer los plazos tanto de un despliegue escalonado de la Agencia en el punto crítico requerido como para la ejecución de las medidas, extremo que no suele ser habitual en la normativa europea en materia de seguridad, ya que sujeta a las Instituciones de la UE y a los Estados miembros a tener que actuar sin demora. En suma, este mecanismo está pensado como «último recurso», ideado a raíz de la última crisis.

La protección de los derechos fundamentales se asegura en el artículo 34 del texto, cuando se establece que los guardias de fronteras están sujetos a la Ley de la Unión en la materia y a las Convenciones internacionales, concretamente la Convención sobre el Estatuto de los Refugiados de 1951, el Protocolo de 1967 y las obligaciones relacionadas con el acceso a la protección internacional, en particular el principio de no devolución.

En los artículos que van desde el 44 al 50 se trata el intercambio de información y la consiguiente protección de datos. En relación con la nueva Agencia, se regula el papel de la base de datos más relevante en la materia, la 
ya expuesta EUROSUR. Además se menciona la necesidad de cooperación entre las distintas agencias europeas involucradas, EUROPOL, EUROJUST y $\mathrm{EASO}^{40}$.

En cuanto a su personal, se estima que podría contar con una plantilla fija de 1.000 empleados -el triple que FRONTEX-, además del contingente de 1.500 agentes cedidos por los Estados miembros y preparados para intervenir en cualquier momento. En concreto, está previsto que España aporte 111 efectivos a la nueva Guardia Costera Europea de Fronteras y Costas ${ }^{41}$.

Por último, destacar que se ha previsto la creación en el seno de la nueva Agencia de una Oficina Europea de Retorno para permitir el despliegue de equipos conjuntos destinados a desarrollar operaciones de retorno. Estos equipos estarían integrados por escoltas, supervisores y especialistas en la materia, con la finalidad de retornar a sus países de origen a los nacionales de terceros Estados en situación irregular. Acompañando a la creación de esta Oficina, se ha previsto la creación de un documento europeo normalizado de viaje para el retorno que debe garantizar una mayor aceptación por parte de los terceros países, según refieren las autoridades europeas.

Es destacable el hecho de que esta regulación ha sido presentada, negociada y aprobada en muy poco tiempo, teniendo en cuenta el ritmo habitual de la UE, sobre todo en lo que a materias de interior se refiere.

\section{CONCLUSIÓN}

Esta última crisis migratoria iniciada en verano de 2015 y considerada por la ONU como «la mayor crisis humanitaria» desde la Segunda Guerra Mundial ha puesto en evidencia a los Estados miembros y a la Unión Europea, en relación con la gestión de las fronteras exteriores, poniendo además en peligro el espacio Schengen, uno de los grandes logros de la Unión.

40 Oficina Europea de Apoyo al Asilo que tiene la misión es una agencia de la Unión Europea (UE) que opera desde 2011, sus funciones son: impulsar la cooperación entre Estados miembros en materia de asilo; apoyar a los Estados miembros cuyos sistemas de asilo y recepción estén sujetos a presiones especiales; mejorar la aplicación del Sistema Europeo Común de Asilo (SECA). Reglamento (UE) n ${ }^{\circ}$ 439/2010 del Parlamento Europeo y del Consejo, de 19 de mayo de 2010, por el que se crea una Oficina Europea de Apoyo al Asilo, DO L 132 de 29.5.2010, p. 11.

41 «España aportará 111 agentes a la nueva Guardia de Fronteras y Costas de la UE», diario $A B C$, edición digital, 08.07.2016, disponible en <www.abc.es> [fecha de consulta: 07.09.2016]. 
Entre los objetivos políticos del ELSJ se encuentra el desarrollo de una política común de inmigración, asilo y control de las fronteras exteriores, es decir que la gestión de estas se configura como una responsabilidad compartida entre los Estados miembros y la Unión Europea. Hasta el momento la UE había implementado ciertos mecanismos de control de las fronteras exteriores dentro del marco del ELSJ, disponiendo de herramientas como bases de datos -el Sistema de Información Schengen y el Sistema de Información de Visados-, la prolífica actividad de la agencia FRONTEX había sido significativa con operaciones de apoyo continuas sobre todo a los países del sur de Europa y el envío de funcionarios de enlace a los países con mayor volumen de entrada. Pero la experiencia revela que en asuntos de interior solo ciertas situaciones desesperadas mueven a los Estados a concluir acuerdos que impliquen un verdadero avance, como sucede en la lucha antiterrorista con los grandes atentados. En este sentido, la última crisis ha supuesto la creación de la Guardia Europea de Costas y Fronteras lo que podría suponer un punto de inflexión en materia de gestión de las fronteras exteriores y por ende en el espacio de libertad, seguridad y justicia. Lo cierto es que aún es pronto para valorar su verdadero alcance, pero sobre el papel es un mecanismo verdaderamente revolucionario en el proceso de integración europea ya que posibilita la actuación directa de la UE sobre el terreno de un Estado miembro. ¿Acaso podría suponer el inicio de un proceso de creación de otros cuerpos europeos de policía en otras materias?

Por otra parte, es evidente que la inmigración no es terrorismo, ni crimen organizado, pero si un medio idóneo para la entrada en suelo europeo de elementos terroristas, por lo que independientemente de la política migratoria del momento debe reclamarse un férreo control en relación con este fenómeno.

En un momento tan sensible para Europa, después de los recientes atentados de origen yihadista es comprensible la preocupación ciudadana y de sus autoridades. El vuelco sucedido en la política interior alemana atribuido a su política migratoria, con punto de partida en un mensaje en Twitter de la Canciller alemana con el texto: «bienvenidos refugiados» es una buena muestra de ello. Se acusa a la Unión Europea de inacción en situaciones de crisis, pero ahora que presenta una mecanismo mediante el que podrá tomar las riendas de una situación fronteriza, ¿cómo será valorado?, ¿como intrusivo o incluso más allá como una maniobra de Alemania para controlar Europa?

Lo cierto es que podría pensarse que fuera una herramienta ideada para intervenir en países como Grecia, que demostraron no estar a la altura de las 
circunstancias en la gestión de la última crisis, pero ¿qué pasaría si se da una situación similar en países de más peso de la UE? ¿Esta impondría su criterio mandando a su Guardia? En fin, Europa no debiera sentirse culpable por el control exhaustivo de sus fronteras exteriores, pero sí comprometida y solidaria con quienes buscan la mejora de sus condiciones de vida o escapar de la violencia en sus Estados de origen; otras vías, como la estabilización de esos Estados, con el compromiso de intervención eficaz en ellos, en colaboración con la comunidad internacional, deben ser también desarrolladas. 